\title{
Uso de Profilaxis Antibiótica en Implantes Dentales: Revisión de la Literatura
}

\author{
Use of Prophylactic Antibiotics in Dental Implants: Literature Review
}

Ramón Fuentes; Benjamín Weber; Tania Flores \& Gonzalo Oporto

FUENTES, R.; WEBER, B.; FLORES, T. \& OPORTO, G. Uso de profilaxis antibiótica en implantes dentales: Revisión de la literatura. Int. J. Odontostomat., 4(1):5-8, 2010.

RESUMEN: Hoy en día la implantología se ha convertido en una excelente alternativa frente a la pérdida o ausencia de piezas dentarias. Todo procedimiento quirúrgico invasivo prevé una bacteremia en la cual un número limitado de especies bacterianas resultan comúnmente implicadas en infecciones post operatorias. Por esta razón, parece imperante definir protocolos a seguir en cuanto al uso correcto de profilaxis antibiótica, ya que, actualmente, mucho de lo que se decide realizar, no se basa en la evidencia científica, sino en las declaraciones anecdóticas de cada uno de los profesionales que las realizan.

PALABRAS CLAVE: profilaxis antibiótica, implantes dentales, bacteremia, antibióticos, complicaciones postoperatorias.

\section{INTRODUCCIÓN}

Hoy en día la implantología se ha convertido en una excelente alternativa frente a la pérdida o ausencia de piezas dentarias. Los costos y beneficios de los tratamientos odontológicos rehabilitadores son significativos para los pacientes, por lo cual la implantología es cada vez más frecuente, llegando a alcanzar en la actualidad tasas de éxito de entre un $95 \%$ a un $99 \%$. Todo procedimiento quirúrgico invasivo prevé una bacteremia en la cual un número limitado de especies bacterianas resultan comúnmente implicadas en infecciones post operatorias (Reference manual, 20082009). El riesgo de contraer infecciones tras una cirugía de implantes depende tanto de la correcta realización de los procedimientos y el buen manejo de los implantes, como del cuidado de mantener vigentes los principios básicos de cirugía y asepsia (Peterson, 1990). Esto ha llevado a la utilización empírica de la profilaxis antibiótica (ABP), que se define como la administración pre o peri operatoria de antibióticos para prevenir una infección local o sistémica y sus consecuencias, a pesar del conflicto existente en los resultados clínicos obtenidos (Dent et al., 1997). En un estudio realizado en 1.544 Odontólogos generales, Palmer et al. (2000), describió que más del $40 \%$ de los odontólogos prescriben antibióticos profilácticos en cirugías orales menores en pacientes sin historia médica que lo amerite con el objetivo de evitar infecciones postoperatorias, siendo la amoxicilina el antibiótico de elección predominante en esta situación. Y por el contrario, entre el $15 \%$ y el $67 \%$ de los odontólogos no prescriben (ABP) en pacientes médicamente comprometidos. Más del $50 \%$ de los odontólogos encuestados requiere solicitar el asesoramiento especializado para determinar el tipo de profilaxis y las indicaciones de su uso y más del $90 \%$ indicó que es recomendable utilizar el actual régimen de profilaxis antibiótica con pacientes con riesgo de endocarditis. Estos resultados sugieren que existe la necesidad imperante de la elaboración de directrices para los profesionales acerca del uso profiláctico de antibióticos. Por su parte, Mazzocchi et al. (2007) sugiere, de acuerdo a los resultados obtenidos en un estudio retrospectivo de 736 implantes sin terapia antibiótica, que la profilaxis antibiótica no provee ventajas para los pacientes. Gynther et al. (1998), comparó dos grupos de pacientes, uno de 147 con terapia profiláctica consistente en la administración de $2 \mathrm{gr}$ de penicilina, 1 hora antes de la cirugía y otro de 132 sin terapia antibiótica, pero en tiempos distintos. Concluyó que una adecuada manipulación de los tejidos du-

` Departamento de Odontología Integral, Universidad de La Frontera, Chile 
rante la cirugía y una buena técnica clínica son, probablemente, factores más importantes en la protección de pacientes a infecciones postoperatorias, que el uso de profilaxis antibiótica, ya que no se hallaron diferencias significativas en la tasa de infección entre el grupo que recibió profilaxis antibiótica y el grupo que no la recibió. Por su parte Esposito et al. (2003) afirman que no se encontraron estudios aleatorios controlados que recomienden criterios antibióticos para prevenir complicaciones tras la cirugía de implantes dentales y concluyó que no existe una adecuada sistemática de pruebas para recomendar o desalentar el uso de la profilaxis con antibióticos para prevenir complicaciones y el fracaso de los mismos. Bowen Antolín et al. (2007) señala que los fracasos de los implantes dentales pueden deberse a: trauma (fuerzas no controladas o mal dirigidas) o infección. Si un implante dental es infectado por bacterias la posibilidad del fracaso del mismo es alto. Por esta razón el uso de profilaxis antibiótica es de aplicación universal. Sin embargo, mucho de lo que se decide realizar en cuanto a profilaxis antibiótica se refiere, actualmente no se basa en la evidencia científica, sino en las declaraciones anecdóticas de cada uno de los profesionales que las realizan. La necesidad del uso de profilaxis antibiótica radica en la elevada tasa de infecciones postoperatorias y en la relación que estas mantienen con importantes efectos nocivos sobre el implante (Lawler et al., 2005). A pesar de ser una práctica controversial, es común la utilización de antibióticos profilácticos o un ciclo de antibióticos tras la colocación del implante, con el objetivo de aumentar las probabilidades de éxito del mismo. Existen estudios observacionales, como el de Dent et al., quien tras analizar 2.641 implantes llegó a la conclusión de que el riesgo de fracaso del implante fue de 2 a 3 veces mayor al no utilizar profilaxis antibiótica, que prueban que el uso de antibioprofilaxis ayuda a reducir la tasa del fracaso temprano de los implantes dentales (Laskin et al., 2000) aunque no se registran ensayos controlados que se hayan llevado a cabo. Las directrices del Colegio Americano de Dentistas sugieren que en cirugías orales maxilofaciales complejas, que incluyen la colocación de implantes dentales, puede resultar beneficiosa la cobertura antibiótica (Laskin et al.) mientras que algunos cirujanos creen que la cobertura antibiótica no es necesaria en las cirugías de colocación de implantes, los continúan usando para evitar reclamos por mala praxis. Este no debería ser el único factor a considerar a la hora de tomar una decisión tan importante como si utilizar o no antibióticos en el ejercicio odontológico (Seymour et al., 2000). Mientras el uso rutinario de antibióticos en el tratamiento de implantes orales todavía parece ser polémico, su uso en cirugía oral y maxilofacial ha sido efi- caz en la prevención de infecciones de la herida postperatoria (Hotz et al., 1994). La profilaxis antibiótica en pacientes sin compromisos médicos no sólo sigue siendo motivo de diferencias en el desarrollo de la práctica clínica (Longman \& Martin, 1991) sino una importante decisión a considerar desde el punto de vista médico-legal para pacientes médicamente comprometidos (Martin et al., 1997). Mientras aproximadamente un tercio de todos los antibióticos utilizados en medicina son prescritos con fines profilácticos (Neu, 1979) la indicación de profilaxis antibiótica antes de una cirugía de implantes no está bien establecida y la correlación del uso de la ABP con fracasos en cirugías de implantes está pobremente documentada (Mazzocchi et al.). Estudios previos señalan, que en la práctica odontológica la prescripción de antibioprofilaxis se ha limitado a la prevención de la endocarditis (Holbrook et al., 1983, 1987). Ampliando un poco el concepto, Esposito et al. agregaron que debe prescribirse para prevenir complicaciones que sean graves amenaza para la vida, como es el caso de pacientes con riesgo de endocarditis, trastornos metabólicos, irradiados en sector de cabeza y cuello, inmunodeficientes y en caso de cirugías de implantes de gran envergadura. Y según Mazzochi et al. para evitar infecciones tras procedimientos quirúrgicos dentoalveolares extensos en pacientes con compromiso general significativo. Muchos odontólogos usan ABP en tratamientos de implantes, sin embargo muchas veces no son utilizados de la forma más eficaz o inclusive no son necesarios. A pesar del hecho de que la incidencia de infecciones asociadas al tratamiento de implantes dentales es muy baja, muchos cirujanos utilizan regímenes de profilaxis antibiótica que se continúan por más de una semana posterior a la instalación del implante (Gynther et al.). Schwartz \& Larson (2007), concluye en su revisión de la literatura, que un significativo porcentaje de agentes antimicrobianos prescritos por dentistas son inapropiados. Reafirma que los dentistas deben considerar como una medida de salud pública el riesgo de sobre utilizar antibióticos incluyendo el riesgo de reacciones alérgicas así como la selección adecuada del antibiótico para evitar crear resistencia a los microorganismos. Plantea que la modificación de la conducta sobre el mal uso de los antibióticos en la odontología se debería afrontar utilizando los últimos protocolos como por ej: la American Heart Association (AHA), monitoreando el uso de antibióticos (AB) por parte de los dentistas, así como una educación continua concerniente a los riesgos de salud pública relacionado con la sobre prescripción de los antibióticos. La importancia de optimizar la terapia antibiótica en la cirugía de implantes, no debe ser descuidada con respecto al evidente riesgo de desarrollar resistencia de algunos de 
los patógenos más comunes en la cavidad oral (Friberg, 1996). Por estas razones, se debe procurar que los beneficios de la ABP se equilibren con los riesgos de reacciones alérgicas, toxicidad, efectos secundarios y el creciente problema de la resistencia antibiótica (World Health Organisation Symposium, 1997; Standing Committee of Science and Technology, 1998). Mientras existen estudios que apoyan la premisa de que los odontólogos podrían estar contribuyendo a la selección de resistencia por el mal uso de antibióticos (Schwartz \& Larson), los principios fundamentales del uso de éstos, a menudo parecen ser ignorados iniciándose, comúnmente en un tiempo inadecuado y extiendiéndose más allá del tiempo indicado (Norris \& Doku, 1992). De la revisión de la literatura se desprende que este riesgo podría reducirse mediante el uso de una única dosis preoperatoria del antibiótico en lugar de múltiples aplicaciones, considerando que el uso prolongado de antibióticos profilácticos en cirugías de implante dental no presentó ventajas o beneficios por sobre el régimen de dosis preoperatoria única (Binahmed et al., 2005). Kashani et al. (2005), agrega que la reducción en el número de dosis de antibiótico a dosis única, sólo el día de la cirugía, no afecta negativamente la tasa de supervivencia de implantes orales y por lo tanto puede ser recomendado por el clínico. El Manual de Referencia de Profilaxis Antibiótica sugiere que un régimen de antibioprofilaxis (ABP) eficaz debe ser administrado poco antes del procedimiento y dirigido contra los microorganismos que tienen mayor probabilidad de infectar al paciente. De la revisión de la literatura se desprende que los antibióticos más usados en $A B P$ son las penicilinas (PNC), siendo el esquema más adecuado de $\mathrm{ABP}$ es el de Peterson, que corresponde a $2 \mathrm{~g}$ de PNC 1 hora antes de la cirugía. Por otro lado, parece imperante la necesidad de definir los términos Antibioprofilaxis y Tratamiento Antibiótico, de esta manera y según Gynther et al., la Antibioprofilaxis corresponde a la dosis preoperatoria y única de un antibiótico con el objetivo de disminuir las posibilidades de infección en un paciente. El tratamiento antibiótico corresponde a las dosis de un antibiótico administradas a un paciente por un número determinado de días, con el objetivo de eliminar un cuadro infeccioso. Por su parte, Fleming \& Newman (1990), concuerda con Gynther et al. en el concepto de tratamiento antibiótico, sin embargo extiende el uso de la antibioprofilaxis a las $24 \mathrm{hrs}$. inmediatamente posteriores a la intervención. Mientras Adell et al. (1985), indica que el uso de $\mathrm{ABP}$ debería extenderse hasta 10 días después de realizada la intervención quirúrgica.

FUENTES, R.; WEBER, B.; FLORES, T. \& OPORTO, G. Use of prophylactic antibiotics in dental implants: Literature review. Int. J. Odontostomat., 4(1):5-8, 2010.

ABSTRACT: Today implantology has become an excellent alternative to the loss or absence of teeth. Any invasive surgical procedure provides a bacteremia in a limited number of bacterial species are commonly involved in post-operative infections. For this reason, it seems to follow prevailing define protocols regarding the proper use of prophylactic antibiotics, currently, much of what is decided, not based on scientific evidence, but anecdotal statements from each of the professionals who perform them.

KEY WORDS: antibiotic prophylaxis, dental implants, bacteremia, antbiotics, postoperative complications.

\section{REFERENCIAS BIBLIOGRÁFICAS}

Adell, R.; Leckholm, U. \& Branemark, P. I. Surgical procedures. In: Branemark, P. I.; Zarb, G. A.; Albrektsson, T. Editor(s). Tissue- integrated prostheses. Chicago, Quintessence Publishing Co. Inc., 1985. pp.211-32.

Binahmed, A.; Stoykewych, A. \& Peterson, L. Single Preoperative Dose Versus Long-term Prophylactic Antibiotic Regimens in Dental Implant Surgery. Int. J. Oral Maxillofac. Implants, 20(1):115-7, 2005.

Bowen Antolín, A.; Pascua García, M. T. \& Nasimi, A. Infections in implantology: from prophylaxis to treatment. Med. Oral Patol. Oral Cir. Bucal, 12(4):E323-30, 2007.

Dent, C. D.; Olson, J. W.; Farish, S. E.; Bellome, J.; Casino, A. J.; Morris, H. F. \& Ochi, S. The influence of preoperative antibiotics on success of endosseous implants up to and including stage II surgery: a study of 2,641 implants. J. Oral Maxillofac. Surg., 55:19-24, 1997.

Esposito, M.; Coulthard, P.; Oliver, R.; Thomsen, P. \& Worthington, $\mathrm{H}$. V. Antibiotics to prevent complications following dental implant treatment. Cochrane Database Syst. Rev., 3:CD004152, 2003. 
Fleming, T. F. \& Newman, M. G. Antimicrobials in implant dentistry. In: Newman, M. G. \& Kornman, K. Antibiotics antimicrobial use in dental practice. Chicago, Quintessence Publishing Co. Inc., 1990. pp.187-200.

Friberg, B. Sterile operating conditions for the placement of intraoral implants. J. Oral Maxillofac. Surg., 54(11):1334-6, 1996.

Gynther, G. W.; Köndell, P. A.; Moberg, L. E. \& Heimdahl, A. Dental implant installation without antibiotic prophylaxis. Oral Surg. Oral Med. Oral Pathol. Oral Radiol. Endod., 85(5):509-11, 1998.

Holbrook, W. P.; Higgins, B. \& Shaw,T. R. Recent changes in antibiotic prophylactic measures taken by dentists against infective endocarditis. J. Antimicrob. Chemother., 20(3):439-46, 1987.

Holbrook, W. P.; Willey, R. F. \& Shaw, T. R. Prophylaxis of infective endocarditis. Br. Dent. J., 154:36-9, 1983.

Hotz, G.; Novotny-Lenhard, J.; Kinzig, M. \& Soergel, F. Single-dose antibiotic prophylaxis in maxillofacial surgery. Chemotherapy, 40(1):65-9, 1994.

Kashani, H.; Dahlin, C. \& Alse'n, B. Influence of different prophylactic antibiotic regimens on implant survival rate: a retrospective clinical study. Clin. Implant Dent. Relat. Res., 7(1):32-5, 2005.

Laskin, D. M.; Dent, C. D.; Morris, H. F.; Ochi, S. \& Olson, J. W. The influence of preoperative antibiotics on success of endosseous implants at 36 months. Ann. Periodontol., 5(1):166-74, 2000.

Lawler, B.; Sambrook, P. J. \& Goss, A. N. Antibiotic prophylaxis for dentoalveolar surgery: is it indicated? Aust. Dent. J., 50:S54-9, 2005.

Longman, L. P. \& Martin, M. V. The use of antibiotics in the prevention of post-operative infection: a reappraisal. Br. Dent. J., 170(7):257-62, 1991.

Martin, M. V.; Butterworth, M. L. \& Longman, L. P. Infective endocarditis and the dental practitioner: a review of 53 cases involving litigation. Br. Dent. J., 182(12):465-8, 1997.

Mazzocchi, A.; Passi, L. \& Moretti, R. Retrospective analysis of 736 implants inserted without antibiotic therapy. J. Oral Maxillofac. Surg., 65(11):2321-3, 2007.
Neu, H. C. Prophylaxis--has it at last come of age? J. Antimicrob. Chemother., 5(4):331-3, 1979.

Norris, L. H. \& Doku, H. C. Antimicrobial prophylaxis in oral surgery. Curr. Opin. Dent., 2:85-92, 1992.

Palmer, N. A.; Pealing, R.; Ireland, R. S. \& Martin, M. V. A study of prophylactic antibiotic prescribing in National Health Service general dental practice in England. Br. Dent. J., 189(1):43-6, 2000.

Peterson, L. Antibiotic prophylaxis against wound infections in oral and maxillofacial surgery. J. Oral Maxillofac. Surg., 48:617-20, 1990.

Reference Manual. Guideline on antibiotic prophylaxis for dental patients at risk for infection. Pediatr. Dent., 30:215-8, 2008-2009.

Schwartz, A. B. \& Larson, E. L. Antibiotic prophylaxis and postoperative complications after tooth extraction and implant placement: a review of the literature. $J$. Dent., 35(12):881-8, 2007.

Seymour, R. A.; Lowry, R.; Whitworth, J. M. \& Martin, M. V. Infective endocarditis, dentistry and antibiotic prophylaxis; time for a rethink? Br. Dent. J., 189(11):610-6, 2000.

Standing Committee of Science and Technology. Resistance to antibiotics and other antimicrobial agents. Great Britain, Parliament, House of Lords, 1998.

World Health Organisation Symposium. Monitoring and management of bacterial resistance to antimicrobial agents: a World Health Organization symposium. Geneva, Switzerland, 29 November-2 December, 1995. Clin. Infect. Dis., 24:S1-176, 1997.

Dirección para correspondencia:

Dr. Ramón Fuentes Femándes

Dpto. de Odontología Integral

Universidad de La Frontera

Manuel Montt 112, Temuco

CHILE.

Email: rfuentes@ufro.cl

Recibido : 14-09-2009

Aceptado: 21-12-2009 